\section{Resúmenes del VII Encuentro Nacional de Medicina del Dormir (agosto 2016)*}

\section{Características video electroencefalográficas: Epilepsia mioclónica progresiva}

Pella-Cruzado VM, Alanis-Guevara I, Pérez-Lara A

Clínica de Epilepsia y Sueño, Ciudad de México

Material y métodos: Estudio retrospectivo, descriptivo a 16 años; se incluyeron pacientes con mioclonia progresiva y monitoreo prolongado (de 24 a 48 h) de video electroencefalograma, se utilizó un equipo Stellate Systems, Harmonie, Sensa. Resultados: Se incluyeron 20 pacientes, 13 mujeres y 7 hombres, edad mínima 8 años, edad máxima de 41 años. 14 pacientes presentaron crisis ictales, $77 \%$ presentó de 2 a 3 tipos de crisis, actividad de fondo Delta y Theta $100 \%$, estado epiléptico 9, $100 \%$ complejo punta onda lenta o polipuntas ondas lentas generalizadas, $80 \%$ punta ondas lentas frontocentrales, $100 \%$ supresión del voltaje posdescarga, 100\% ondas lentas de alto voltaje, estado epiléptico eléctrico durante el sueño 6 pacientes. Conclusiones: La presencia de complejos punta onda lenta y polipuntas ondas lentas generalizadas presididas de supresión del voltaje que se exacerban durante el sueño, son característicos de la epilepsia miocló- nica progresiva independientemente de la causa y evolución.

\section{Prevalencia de glaucoma en pacientes con síndrome de apnea obstructiva de sueño}

Mancera-Leyva OE, * Haro-Valencia $R, \neq$ Labra-Herrera $A, \neq$ Gutiérrez-Carreón A*

*Universidad Autónoma de Baja California, ‡Universidad Nacional Autónoma de México

Introducción: El glaucoma presenta una prevalencia del $3.74 \%$ en la población general. Se ha sugerido la relación causa-efecto del síndrome de apnea obstructiva de sueño con trastornos como hipertensión, coagulopatías e incluso con glaucoma, este último con una prevalencia que puede alcanzar el $27 \%$ en pacientes con apnea obstructiva de sueño. Se ha sugerido la detención de la evolución del glaucoma al tratar exitosamente el síndrome de apnea obstructiva de sueño en pacientes que padecen ambos trastornos. Hipótesis: La prevalencia de glaucoma es mayor en pacientes con síndrome de apnea obstructiva de sueño que en la población general. Objetivo: Conocer la prevalencia de glaucoma en pacientes con síndrome de apnea obstructiva de sueño que son atendidos en el Instituto Mexicano de Medicina Integral de Sueño en la Ciudad de México en el año 2015. Material y métodos: La información se obtuvo mediante la extracción de datos a partir de los expedientes clínicos de los pacientes atendidos en Instituto Mexicano de Medicina Integral de Sueño, Ciudad de formación se llevó a cabo mediante tabulaciones usando el software Microsoft Excel. Se realizó la comparación de la cantidad México en el año 2015. El registro de la in-

\footnotetext{
* La transcripción de los resúmenes es responsabilidad de la Academia Mexicana de Medicina del Dormir, así como en el orden que aparecen.
}

de pacientes con diagnóstico de síndrome de apnea obstructiva de sueño y glaucoma, con los que sólo presentaron síndrome de apnea obstructiva de sueño. Resultados: 2 de 62 pacientes $(3.2 \%)$ con síndrome de apnea obstructiva de sueño también presentaron glaucoma. Conclusiones: En la población estudiada, la prevalencia de glaucoma en pacientes con síndrome de apnea obstructiva del sueño resultó menor en comparación con la población general, sugiriendo que no hay relación causa-efecto entre ambos trastornos.

\section{Terapia cognitivo conductual para el insomnio en pacientes con insomnio asociado a un episodio depresivo (trabajo ganador a la mejor presentación oral)}

Tena-Razo A, Jiménez-Genchi A
Instituto Nacional de Psiquiatría

Introducción: En pacientes deprimidos, el insomnio aumenta el riesgo de conductas suicidas, recaídas y recurrencias. Habitualmente se utilizan agonistas de los receptores de benzodiacepinas para el tratamiento del insomnio. Una desventaja son los efectos adversos. Una alternativa es la terapia cognitivo conductual para el insomnio (TCC-I), la cual ha demostrado ser eficaz en insomnio primario, con pocos estudios en insomnio asociado a depresión. Hipótesis: La TCC-I genera mejorías del insomnio en pacientes deprimidos. Estas mejorías son mayores que las obtenidas sólo con tratamiento farmacológico. Objetivo: Establecer los beneficios de adicionar al tratamiento farmacológico, un programa de TCC-I en pacientes con insomnio y depresión. Material y métodos: Incluimos 
pacientes ambulatorios con depresión e insomnio recibiendo farmacoterapia de acuerdo con las preferencias de su médico. Se formaron dos grupos. El primer grupo recibió un programa de cuatro sesiones de TCC-I. En cada sesión se incluyó una intervención diferente (información básica sobre sueño, higiene de sueño, control de estímulos, restricción del tiempo en cama y terapia cognitiva). El segundo grupo funcionó como control. La evaluación de seguimiento (Índice de Severidad del Insomnio -ISI- y Escala de Depresión de Hamilton) se realizó al finalizar las 4 sesiones en el grupo de terapia y posterior a un mes en los controles. Resultados: Se asignaron 20 y 15 pacientes al grupo de terapia y control, respectivamente. En comparación con el grupo control, los pacientes que recibieron la terapia presentaron una mejoría significativamente mayor en las puntuaciones totales del ISI (7.65 puntos en el grupo de terapia $v s .2 .4$ puntos en controles). Se observó una tendencia a una mayor reducción de la gravedad de la depresión en el grupo de terapia. Conclusiones: El presente ensayo clínico demostró la eficacia a corto plazo de la TCC-I para el tratamiento del insomnio en adultos con depresión.

\section{Determinación de} marcadores del ciclo sueño-vigilia en pacientes diagnosticados con hipersomnias de origen central

\section{Ayala-García JC, Velázquez- Moctezuma J, Arana-Lechuga DY, Terán-Pérez GJ \\ Universidad Autónoma \\ Metropolitana-Iztapalapa}

Introducción: Existe una correlación importante entre la actividad sueño-vigilia y la secreción de diversas moléculas. Estas correlaciones se han atribuido en parte, a que existe una estrecha relación y sincronización circadiana entre la secreción de estas moléculas y el sueño. Estas observaciones han sido de gran importancia, pero aún hoy en día se desconoce en gran medida los mecanismos por los cuales se presenta la somnolencia excesiva diurna en los pacientes que presentan hipersomnias de origen central. Hipótesis: Pacientes diagnosticados con narcolepsia o hipersomnia idiopática sin problemas agregados de salud presentarán alteraciones en las concentraciones plasmáticas de citocinas, melatonina, cortisol, adipocinas, TSH y parámetros metabólicos con respecto a sujetos de menor riesgo. Objetivo: Cuantificar adipocinas, citocinas, melatonina, cortisol, TSH y marcadores metabólicos en pacientes con hipersomnias de origen central. Material y métodos: Se obtuvieron muestras de sangre y saliva en 11 pacientes diagnosticados con narcolepsia e hipersomnia idiopática, fueron pareados por edad y sexo con sujetos de menor riesgo. Se midió glucosa, colesterol y triglicéridos mediante fotometría de reflectancia. Las concentraciones de grelina, leptina, TNF- $\alpha$, TSH, melatonina y cortisol fueron cuantificadas por duplicado mediante la técnica de ELISA. Las muestras fueron procesadas de acuerdo con las especificaciones de cada KIT comercial y medidas mediante espectrofotometría. Resultados: Observamos que las concentraciones de leptina plasmática y colesterol en sangre, son significativamente distintas a las presentadas por los sujetos control. Conclusión: La leptina podría ser una de las moléculas importantes en el mecanismo que genera la somnolencia excesiva diurna que presentan las personas diagnosticadas con hipersomnia idiopática.

\section{Evaluación de nuevo dispositivo de avance mandibular como tratamiento en pacientes con SAOS y ronquido}

\section{Cortés-Mejía JM, Arana-Lechuga DY, Velázquez-Moctezuma J, Terán-Pére GJ, Esquéda-León E}

Universidad Autónoma Metropolitana

Introducción: El síndrome de apnea obstructiva sueño (SAOS) está caracterizado por períodos de obstrucción completa (apnea) o parcial (hipoapnea) de la vía aérea alta provocando una desaturación de oxígeno en la sangre que concluye con breves alertamientos durante el sueño. La somnolencia excesiva diurna es el síntoma principal. El paciente presenta un sueño no reparador afectando en gran medida su rendimiento laboral, intelectual, físico, cognitivo y es directamente asociado con accidentes vehiculares. Uno de los tratamientos más estudiados, desarrollados y aceptados en los últimos años para el SAOS es el uso de dispositivos de avance mandibular (DAM). En México se utilizan mayormente dispositivos importados y prefabricados lo que complica el apego y el acceso al tratamiento. Hipótesis: El dispositivo de avance mandibuar de avance gradual y personalizado será eficaz, cómodo y de fácil acceso para el tratamiento de SAOS y ronquido leve a moderado. Objetivo: Evaluar la calidad de sueño en los pacientes antes y después de utilizar un nuevo dispositivo de avance mandibular personalizado fabricado en México con técnicas y materiales de uso dental. Material y métodos: Se reclutaron 50 pacientes diagnosticados por medio de polisomnografía con ronquido primario y SAOS leve y moderado ambos géneros de 18 años y hasta 60. Se fabricaron los dispositivos personalizados y se realizó un segundo estudio polisomnográfico durmiendo con el dispositivo. Se evaluaron las variables de calidad de sueño objetivas y subjetivas pre y postratamiento. Se realizaron tomografías axiales computarizadas a 20 pacientes con y sin DAM colocado en boca para evaluar el calibre de la vía aérea. Resultados: Se encontraron diferencias significativas en las siguientes variables: hipoapneas, índice apnea hipoapnea, apnea obstructiva, desaturaciones de oxígeno, somnolencia diurna y ronquido. Conclusiones: El dispositivo de avance mandibular disminuye el IAH, somnolencia diurna y el ronquido significativamente.

Una mascarilla personalizada para suministrar presión positiva en pacientes neuromusculares con trastornos respiratorio del dormir 


\section{Ramírez-Molina VR, Carrillo-Alduenda $J$, * Urrusti-Alonso JL,, Buzali-Wlodaver I $^{\ddagger}$}

*Instituto Nacional de Enfermedades Respiratorias Ismael Cosío Villegas, ‡Universidad Iberoamericana

Introducción: El tratamiento de los trastornos respiratorios del dormir (TRD) en presencia de enfermedad neuromuscular (ENM) es un dispositivo de presión positiva durante el sueño. Los pacientes con ENM muy frecuentemente tienen hipotrofia muscular, desnutrición y dismorfias craneofaciales que dificultan encontrar una mascarilla que se ajuste adecuadamente a la piel de la cara. Actualmente existen impresores 3D que podrían fabricar una mascarilla personalizada y que podrían resolver este problema. Objetivos: Describir el proceso para desarrollar una mascarilla personalizada para suministrar presión positiva en pacientes con TRD y ENM; describir el apego, fuga y efectos adversos a un dispositivo de presión positiva usando una mascarilla personalizada en pacientes con TRD y ENM en una prueba de 1 semana. Material y métodos: Estudio observacional y descriptivo. Criterios de inclusión: mayores de 18 años de edad, género indistinto, con enfermedad neuromuscular e indicación de tratamiento con dispositivo de presión positiva, firma de consentimiento informado. Resultados: Se fabricó una mascarilla personalizada por cada uno de los diez pacientes reclutados con el siguiente procedimiento: réplicas faciales, escaneado 3D de la réplica facial, procesamiento de la imagen 3D, diseño de la mascarilla, impresión de la mascarilla, ensamble del arnés, válvula en mascarilla y sello antifugas, pruebas de la mascarilla, resultados de la prueba. No se encontró diferencias en apego, fuga, molestias y beneficios con respecto a un grupo de controles históricos. Conclusiones: Este proyecto demuestra la factibilidad de fabricar mascarillas personalizadas para administrar presión positiva en las vías aéreas de pacientes con trastorno respiratorio del dormir y enfermedad neuromuscular, con buena comodidad y desempeño.

\section{Actividad cognitiva pre sueño en pacientes con insomnio, depresión mayor e insomnio y buenos durmientes}

\section{Saldívar-Hernández RK, Jiménez-Genchi $A$}

Instituto Nacional de Psiquiatría

«Ramón de la Fuente Muñiz»

Introducción: La actividad cognitiva pre sueño (ACPS) entendida como las preocupaciones, pensamientos intrusivos y una mente hiperactiva previa al dormir, actúa como precipitante y/o perpetuador del insomnio. Considerando la elevada comorbilidad entre la depresión mayor (DM) y el trastorno por insomnio (TI) es importante saber si la ACPS opera de manera similar cuando se presentan ambos padecimientos y si guarda relación con la práctica de las medidas de higiene de sueño (PMHS). Hipótesis: Los individuos con TI y con depresión mayor e insomnio (DMI) tendrán mayor ACPS y menor calidad de sueño que los buenos durmientes (BD), la cual se asociará con la PMHS. Objetivos: Evaluar la ACPS en pacientes con TI, DMI y BD y su asociación con la PMHS. Material y métodos: Muestra de 19 sujetos con TI, 19 con DMI y 19 BD. El diagnóstico se estableció mediante la entrevista neuropsiquiátrica internacional; para evaluar las variables de interés se emplearon: escala de activación pre sueño, escala de prácticas de higiene de sueño. Se empleó ANOVA de una vía y coeficiente de correlación de Pearson. Resultados: Los sujetos con DMI mostraron significativamente más ACPS (47.8 $\pm 10.5)$ que aquéllos con TI (31.89 \pm 9.0$)$ y éstos a su vez más que los BD (20.47 \pm 3.2) (F 53.3, gl 2, p < .001). La ACPS se relacionó significativamente con la PMHS solamente en los BD ( $r=0.53$, p 0.019), específicamente con las conductas de regulación homeostática y/o circadiana ( $\mathrm{r}$ $=0.49, \mathrm{p} 0.32)$ y de alertamiento $(r=0.67$, $\mathrm{p}=0.02)$. Sin embargo, los componentes somático y cognitivo sólo se relacionaron de manera significativa con conductas de alertamiento $(r=0.52$ y $r=0.62, p$
$<0.05)$. Conclusiones: Los sujetos con DMI presentan más ACPS que los sujetos con TI; sin embargo, en estos grupos la ACPS no parece estar influenciada por las PMHS, mientras que en BD, las conductas de alertamiento de las PMHS se asocian con más ACPS.

\section{Efectos del turno rotatorio laboral en pacientes con síndrome de apnea obstructiva de sueño}

\section{Verde-Tinoco IS, Santana-Miranda $R$, Gutiérrez-Escobar R, Jiménez-Correa $U$}

Clínica de Trastornos del Sueño, UNAM

Introducción: La adaptación a nuevos ciclos laborales implica disminuir en promedio cuatro horas menos el tiempo total de sueño habitual, resultando ser un sueño insuficiente. Si además se padece de un trastorno respiratorio durante el dormir, el sueño será fragmentado por la presencia de despertares asociados a los eventos respiratorios, aunado al impacto sobre la arquitectura del dormir. Ambas situaciones se asocian de manera independiente a incremento de alteraciones metabólicas, riesgo cardiovascular, riesgo de accidentes, bajo rendimiento y disminución de la calidad de vida. Objetivo: Comparar la calidad subjetiva del sueño y de las variables polisomnográficas de población con SAOS con turno rotatorio de trabajo y turno diurno. Material y métodos: Se realizó un estudio retrospectivo comparativo con una muestra de 107 sujetos masculinos, distribuidos en tres grupos: grupo A conformado por sujetos con SAOS de intensidad severo con turno de trabajo diurno $(n=45,46$ años \pm e.e. 1.6), grupo B conformado por sujetos con SAOS severa con turno rotatorio de trabajo $(n=45,49$ años \pm e.e. 1.1$)$ y grupo $\mathrm{C}$ sujetos control $(\mathrm{n}=17,33$ años \pm e.e. 2.2). Los participantes fueron evaluados mediante batería de pruebas de calidad subjetiva del sueño, nivel de somnolencia y estudio polisomnográfico en la Clínica de Trastornos del Sueño de la UNAM. Se aplicó prueba de análisis de varianza 
(ANOVA) de una vía para comparar las variables relacionadas con la calidad subjetiva de sueño y arquitectura de sueño entre los tres grupos; además un análisis post hoc de Tukey para determinar la fuente significancia de $\mathrm{p} \leq 0.05$. Resultados: La valoración de la arquitectura de sueño entre los sujetos con SAOS no mostró diferencias; sin embargo, se determinaron diferencias estadísticamente significativas en variables respiratorias, microdespertares y valoración subjetiva del sueño entre estos sujetos, asociado a una diferencia en el número de horas de sueño (NHS). Conclusiones: Con los resultados de este trabajo se cuenta con evidencia para plantear la hipótesis de que la restricción de sueño podría ser un factor que aminore los efectos de los trastornos respiratorios durante el dormir, por evitar la sobreexposición a la hipoxia intermitente de individuos con SAOS con mayor número de horas de sueño. Los efectos de la restricción de sueño por trabajo en turno rotatorio y presencia de SAOS requieren de un interés particular de futuras investigaciones.

\section{Calidad de sueño en estudiantes universitarios: diferencias entre el uso de la cama y hamaca}

\section{Saenz-Cabrera ME, Gonzáles- Gonzáles RS, Gongora-Martín JA, Flores-Escalante DL}

Universidad Autónoma de Yucatán

Introducción: Investigaciones indican que el lugar donde se duerme repercute en la calidad de sueño. En general, los datos sugieren que la cama es el lugar indicado para dormir; sin embargo, la hamaca y su comodidad se piensa que puede llegar a ser mejor debido a que es de uso común en culturas como el Sur de México o el norte de Brasil. Por ejemplo, en Yucatán, Mex., el 59\% de los escolares y el $40 \%$ de los universitarios duermen en hamaca. Hipótesis: HO no existen diferencias estadísticamente significativas entre la calidad de sueño de los estudiantes universitarios y el lugar en donde duermen. Objetivo:
Comparar la calidad de sueño entre los estudiantes que duermen en cama y hamaca. Resultados: La muestra total fue de 254 estudiantes, con una media de edad de 20.55 y una desviación estándar de 1.55. El presente estudio fue abordado mediante un enfoque cuantitativo con diseño descriptivo. Se aplicaron dos instrumentos, el primero fue sobre los hábitos de sueño y el segundo sobre insomnio. Se encontró que el $40.3 \%$ (102) menciona dormir «bien», $52.2 \%$ (132) indica dormir de una manera «regular» y el 7.5\% (19) menciona dormir «mal». De igual forma, y al realizar una prueba t de Student para muestras independientes se obtuvo una $\mathrm{p}=.708$, lo cual indica que se acepta la hipótesis nula. Conclusiones: Los estudiantes universitarios de esta población consideran que el lugar donde duermen (cama o hamaca) no es un factor determinante para la calidad de sueño, sin embargo, es probable que se necesite explorar más sobre el tema para verificar si los resultados son consistentes en distintas poblaciones.

\section{Estimulación eléctrica craneal sobre los síntomas de insomnio, depresión y ansiedad (mención honorífica)}

\section{Moo-Estrella J, Buenfíl-Hernández Al, Pérez-Pichardo MF, * Freyre-Vega CA, Rodríguez-Cámara AC, Carrillo-Sulub AM}

Universidad Autónoma de Yucatán

Introducción: El insomnio es una alteración del sueño que afecta al $30 \%$ de la población, una alternativa de tratamiento propuesta es la estimulación eléctrica craneal (EEC) que consisten en una técnica terapéutica no invasiva que envía pulsos de corriente eléctrica $(<4 \mathrm{~mA})$ al cerebro a través de electrodos. Sin embargo, los alcances de la EEG no son claros así como su relación con los síntomas de ansiedad y depresión. Objetivo: Conocer el efecto de la EEC como tratamiento de los síntomas de insomnio, ansiedad y depresión. Método: Se incluyen 20 sujetos de consulta de primera vez que cumplieron los criterios de insomnio de acuerdo a la ISDC-2, 55\% fueron mujeres, con una edad promedio de 32.10 ( \pm 14.24) años, distribuidos equitativamente entre grupo control y experimental. Los instrumentos empleados fueron el Inventario de depresión Beck (BDI), el cuestionario de ansiedad-estado (CAE) de Spielberger y el índice de gravedad de insomnio (ISI). Se usó el dispositivo de EEC Fisher Wallace, modelo FW 100, el cual se les entregó a cada sujeto por 10 días, y fue empleado durante 20 minutos cada noche antes de dormir y 20 minutos en la mañana al despertarse. Los cuestionarios fueron aplicados antes y después del tratamiento. Resultados: Se encontraron diferencias entre pre y pos del grupo experimental en el ISI (M $=17.5 \pm 4.0 v s . \mathrm{M}=11.1 \pm 5.5), \mathrm{CAE}(\mathrm{M}$ $=43.8 \pm 12.5$ vs. $\mathrm{M}=33.1 \pm 8.3) \mathrm{y}$ BDI $(\mathrm{M}=12 \pm 6.2$ vs. $\mathrm{M}=5.1 \pm 2.8) \mathrm{y}$ en el grupo control sólo se encontró efecto en los síntomas de insomnio $(\mathrm{M}=18.6 \pm 4.5$ vs. $\mathrm{M}=13.1 \pm 8.6)$ y depresión $(\mathrm{M}=14.3$ \pm 5.4 vs. $\mathrm{M}=9.5 \pm 8.1$ ). Conclusiones: La EEC mostró mayores diferencias en el grupo experimental, quizás sus efectos sean mayores sobre los síntomas de ansiedad con repercusiones positivas sobre los síntomas de insomnio y depresión.

\section{Funciones cognitivas entre escolares con sobrepeso/obesidad y trastornos respiratorios durante el sueño}

\section{Martínez-Yáñez G, Valázquez- Moctezuma J, Arana-Lechuga DY}

Clínica de Trastornos del Sueño de la Universidad Autónoma Metropolitana Iztapalapa

Introducción: La obesidad infantil es uno de los problemas de salud pública más graves del siglo XXI. De acuerdo con la Organización Mundial de la Salud (OMS), la obesidad se define como la acumulación anormal o excesiva de grasa. En México, 5,664,870 niños de entre 5 y 11 años de edad presentan exceso de peso corporal (sobrepeso u obesidad) (ENSANUT, 2012). No obstante con ser 
un problema propio de salud, es un factor predisponente de otras enfermedades incluidos los trastornos respiratorios durante el sueño (TRS). De acuerdo con la Clasificación Internacional de los Trastornos del Sueño (ICSD-3), uno de los principales trastornos del sueño es la apnea obstructiva del sueño (AOS), que se caracteriza por la obstrucción parcial o completa e intermitente o prolongada de la vía aérea superior que interrumpe la ventilación normal durante el sueño, patrones normales del sueño o ambos. Diversos estudios han reportado que la obesidad genera deterioro cognitivo. A su vez, la mala calidad de sueño y los TRS también propician el decremento del desempeño cognitivo. Objetivo: Describir las diferencias en el rendimiento cognitivo en niños escolares de 6 a 11 años de edad con sobrepeso/obesidad y TRS. Hipótesis: Se espera encontrar un menor rendimiento cognitivo en el grupo de niños escolares con sobrepeso/obesidad y AOS, respecto al grupo de niños escolares con sobrepeso/obesidad sin TRS y respecto al grupo de bajo riesgo. Material y métodos: Se evaluaron neuropsicológicamente y polisomnográficamente 33 niños escolares, 14 niñas y 19 niños de 6 a 11 años de edad con y sin sobrepeso/obesidad y con y sin TRS. La evaluación neuropsicológica se llevó a cabo con el NEUROPSI atención y memoria, mientras que la evaluación polisomnográfica fue realizada con base en el sistema internacional 10-20. Resultados: En la comparación del rendimiento cognitivo entre grupos, se encontró un desempeño menor en el grupo con presencia de sobrepeso/obesidad y TRS respecto a los demás grupos; sin embargo, los puntajes obtenidos se encuentran dentro del rango de normalidad para los cuatro grupos evaluados. Conclusiones: Se corrobora el marco teórico sobre relación de la obesidad y deterioro cognitivo sin importar la presencia o ausencia de TRS. No se corrobora el marco teórico sobre la relación de los TRS y deterioro cognitivo sin importar la presencia o ausencia de sobrepeso/obesidad.

\section{Prevalencia de eritrocitosis en}

pacientes adultos con apnea obstructiva del sueño en población mexicana

Romero-López Z, Colonia-Cano CJ,
Ochoa-Vázquez MD, Gracia-Ramos
AE, Mata-Marín JA

Centro Médico Nacional «La Raza», IMSS

Objetivo: Determinar la prevalencia de eritrocitosis en pacientes con síndrome de apnea obstructiva del sueño atendidos en el Hospital General Dr. Gaudencio González Garza del Centro Médico Nacional «La Raza» en el período comprendido entre 1 de abril al 1 de julio de 2016. Material y métodos: Se incluyeron pacientes con síndrome de apnea obstructiva de sueño que fueron diagnosticados en la Clínica de Sueño del Centro Médico Nacional «La Raza», se analizaron según el grado de SAOS y el grado de obesidad. La descripción general de los resultados se realizó mediante medidas de tendencia central y de dispersión. Resultados: De una muestra de 227 pacientes se encontró una prevalencia de eritrocitosis en pacientes con SAOS del 39.6\% (IC 95\%, 0.330.46). Los hombres presentaron una mayor prevalencia de eritrocitosis. Los pacientes con SAOS grave presentaron una prevalencia del 29\% (IC 95\%, 0.23-0.35). Los pacientes con obesidad presentaron una prevalencia del $38.7 \%$ (IC 95\%, 0.32-0.45) y fue mayor en los pacientes con obesidad grado III. Se encontró una correlación fuerte con la presencia de eritrocitosis y los niveles de hemoglobina y eritrocitosis. Conclusiones: La prevalencia de eritrocitosis en pacientes con síndrome de apnea obstructiva del sueño es mayor que la reportada previamente en la literatura. Siendo más prevalente en pacientes con diagnóstico de obesidad y en aquéllos pacientes con SAOS grave.
Validación de un cuestionario predictor de apego a CPAP en pacientes con apnea del sueño

Romero-López Z, Soto-Vidal G, Ochoa-Vázquez MD, Gracia-Ramos $A E$, Mata-Marín JA

*Centro Médico Nacional «La Raza», IMSS

Introducción: El éxito en el tratamiento de la apnea obstructiva del sueño (AOS) radica en el apego terapéutico. El uso de cuestionarios para predecir apego a la presión positiva continua en la vía aérea (CPAP) se fundamenta en que la experiencia obtenida, el primer contacto con CPAP es excelente predictor del uso continuado y permite de forma temprana la toma de decisiones para una adecuada constancia en el tratamiento. Objetivo: Construir, aplicar y validar un cuestionario predictor del apego a CPAP en pacientes adultos con diagnóstico reciente de AOS en la Clínica del Sueño del Departamento de Neumología del Hospital General Centro Médico Nacional «La Raza». Material y métodos: Estudio transversal, prolectivo, observacional. El diseño y validación del cuestionario se realizó por multimétodo. La validez de contenido requirió un panel de expertos. Obtenido el cuestionario se evaluó consistencia interna y re-test en un grupo piloto. Posteriormente se aplicó a 124 pacientes tras la noche de titulación de CPAP. La validez de constructo se efectuó comparando las respuestas con la lectura de dispositivo de memoria a 30 días de uso. Finalmente se obtuvo sensibilidad, especificidad, VPP, VPN. Resultados: Tras el análisis final, de un total de 14 preguntas, 4 obtuvieron $\mathrm{p}<$ 0.05 para el apego a CPAP, pregunta 1 sensibilidad $86.3 \%$, VPN $80 \%$; pregunta 2 sensibilidad $81.8 \%$; VPN $76.4 \%$; pregunta 7 sensibilidad 77.2\%; VPN 79.1\%; pregunta 9 sensibilidad $72.7 \%$; VPN 71.4\%. Conclusión: El cuestionario diseñado para predecir el apego a CPAP en pacientes con AOS resultó ser válido y confiable como predictor de apego.

\section{Fármacos antidepresivos para síntomas en narcolepsia. Revisión sistemática de la literatura}




\section{(trabajo ganador a la mejor presentación en póster)}

\author{
Williams-De Roux $R$, * \\ Portillo-Vázquez AM, ${ }^{\ddagger}$ Torres-Fraga MG
}

*Instituto Nacional de Ciencias Médicas y Nutrición «Salvador Zubirán», ‡Instituto Nacional de Enfermedades Respiratorias Ismael Cosío Villegas

Introducción: La narcolepsia es una enfermedad neurológica que genera un alto impacto sobre la salud de quien la padece ya que cursa con síntomas con frecuencia discapacitantes, como la somnolencia excesiva diurna y la cataplejía, que es una súbita pérdida del tono muscular; ambos síntomas predisponen a un mayor riesgo de accidentes. El tratamiento de la narcolepsia tiene como objetivo mejorar la somnolencia excesiva diurna y controlar los eventos de cataplejía y para ello se han utilizado diferentes grupos de fármacos, de los cuales los más disponibles y económicos son los antidepresivos. Material y métodos: Se realizó una búsqueda sistematizada en 9 bases de datos: Medline, Koreamed, Central, LILACs, African Index Medicus, Embase, WHO, Openthesis, PsycInfo. Para la búsqueda de ensayos no publicados se realizó búsqueda en CENTRAL. Se incluyeron ensayos clínicos controlados, paralelos o cruzados, aleatorizados que compararan el uso de antidepresivo en el tratamiento de narcolepsia $v s$. placebo o vs. otro antidepresivo. La extracción de los datos y la evaluación de la calidad de los estudios fue realizada por dos investigadores en forma independiente. Resultados: Se incluyeron 5 estudios, con un total de 246 participantes. En 4 de ellos se compararon antidepresivos contra placebo, usando 3 diferentes fármacos, los 2 que evaluaron ritanserina, lo hicieron a diferentes dosis y con diferentes escalas de medición. Un quinto estudio comparó 2 diferentes antidepresivos tricíclicos. El riesgo de sesgo en general fue poco claro en la mayoría de los trabajos. El efecto de los antidepresivos sobre la somnolencia excesiva diurna no se ha podido demostrar cuando se mide en forma objetiva por medio de latencias múltiples de sueño y sólo se reporta un discreto efecto sobre la cataplejía en 2 de los trabajos revisados. Conclusiones: La evidencia actual no permite concluir un efecto de los antidepresivos sobre la somnolencia excesiva diurna y la cataplejía en pacientes con narcolepsia.

\section{Efecto sobre el sueño de la primera noche en pacientes con trastornos del espectro autista}

\section{Ayala-Guerrero F, Mateos-Salgado EL, Cebreros-Paniagua R, García-Vite T}

Facultad de Psicología, UNAM

Introducción: El efecto sobre el sueño de la primera noche se refiere a una alteración de los patrones regulares del sueño obtenidos durante un estudio polisomnográfico, por lo que es recomendable registrar cuando menos 2 noches consecutivas, considerando la primera como noche de habituación. En pacientes con el trastorno del espectro autista (TEA) se presenta una alta prevalencia de problemas de sueño. Sin embargo, no se ha analizado adecuadamente el efecto de la primera noche sobre el sueño. Uno de los factores que influyen en la presencia del efecto de la primera noche es la capacidad de las personas de adaptarse a dormir en lugares no familiares, lo que con frecuencia induce estrés y altera su sueño. Dentro de este contexto consideramos pertinente analizar la capacidad de adaptación de los pacientes con TEA para dormir en escenarios no habituales. Hipótesis: Los pacientes con TEA presentarán efectos sobre el sueño de la primera noche significativamente mayores que los participantes control. Objetivo: Comparar las características del efecto sobre el sueño de la primera noche en pacientes con TEA con un grupo control sano. Material y métodos: Equipo Cadwell Esay II, 14 pacientes del sexo masculino con diagnóstico de síndrome de Asperger con una edad de $10.1( \pm 3.3)$ años y 14 controles sanos con 9.7 ( \pm 2.6$)$ años de edad del mismo sexo. Se realizaron registros polisomnográficos durante 2 noches consecutivas. La calificación de las fases de sueño se realizó de acuerdo con las normas de la AASM. Resultados: Tanto los pacientes como los controles mostraron el efecto sobre el sueño de la primera noche, ya que la calidad de su sueño mejoró durante la segunda noche. Sin embargo, el efecto fue significativamente mayor en los pacientes con TEA. Conclusiones: El efecto sobre el sueño de la primera noche es más marcado en pacientes con TEA que en sujetos sanos.

\section{Caracterización del ritmo «Mu» en el sueño de niños con el trastorno del espectro autista (mención honorífica)}

\section{García-Vite TK, Ayala-Guerrero F, Mateos-Salgado E, Cebreros-Paniagua $R$}

Laboratorio de Neurociencias, Facultad de Psicología, UNAM

Introducción: El trastorno del espectro autista (TEA) se caracteriza por dificultades en la comunicación, interacción social y la presencia de comportamientos estereotipados. La teoría de la mente explica el autismo vinculando las deficiencias sociales con una dificultad para representar los estados mentales de otros. Se ha buscado un posible fundamento neuronal relacionado con la habilidad para entender el estado mental del otro estudiando la activación de regiones cerebrales indispensables para la representación de dichos estados. Dado que la facilidad para la activación de dichas regiones está basada en la simulación de acciones observadas en otros, se le ha dado especial interés al ritmo $\mathrm{Mu}$, el cual está dentro del rango de frecuencia de 8-13 hz. Este ritmo tiende a atenuarse con estimulación motora, ya sea observada, ejecutada o imaginada. En pacientes con TEA la reactividad fisiológica a eventos motores del ritmo Mu durante la vigilia se ve afectada; lo cual es un reflejo, de acuerdo con ciertos autores de los problemas de comunicación y socialización que presentan personas con este trastorno. Objetivos: Dado que durante el sueño hay fenómenos relacionados con el procesamiento sensomotor, el propósito de este estudio fue caracteri- 
zar el ritmo Mu en el sueño de niños con TEA y compararlo con un grupo control. Material y métodos: Se llevaron a cabo registros polisomnográficos durante 2 noches consecutivas en cada participante. Se analizaron visual y cuantitativamente los registros. Resultados: Se observó una mayor cantidad de ritmo Mu durante la fase N2 de sueño en ambos. Sin embargo, fue más abundante en el grupo control. Lo anterior coincide con estudios hechos en vigilia. Conclusiones: Se sugiere que el ritmo $\mathrm{Mu}$ participa en procesos de socialización.

\section{Factores antropométricos asociados con la severidad del índice de apnea e hipopnea en la población mexicana}

\section{Ruiz-Morales M, Cortés-Benavides MC, Millán-Rosas G, Santana-Vargas D, Mancilla-Hernández V, Jiménez-} Correa U

Clínica de Trastornos del Sueño, Facultad de Medicina, UNAM

Introducción: En el síndrome de apneahipopnea obstructiva del sueño (SAHOS), clínicamente el perímetro del cuello (PC) ha sido conocido por ser un fuerte predictor del SAHOS. Sin embargo, algunos autores han demostrado que el IMC se correlaciona más con el SAHOS que la circunferencia del cuello. Objetivos: Valorar cuáles son los parámetros antropométricos que actualmente se asocian con la severidad del índice de apnea hipopnea (IAH) en la población mexicana debido a las características antropométricas de nuestra población. Material y métodos: Estudio retrospectivo en el que se compararon los parámetros antropométricos obtenidos de 252 expedientes de pacientes del área de trastornos respiratorios de la Clínica de Trastornos del Sueño de la UNAM, en el período comprendido de enero a diciembre del año 2014. Se utilizó un análisis de varianza de una vía para determinar la fuente de la significancia. Se tomó como valor significativo una $\mathrm{p}$ $<0.05$ Resultados: En cuanto al análisis de varianza de una vía se identificó un incremento significativo en el IMC ( F = 5.34, $\mathrm{p}<.001$ ), el perímetro del cuello ( $\mathrm{F}$ $=3.62, \mathrm{p}<.016)$, Epworth $(\mathrm{F}=4.92, \mathrm{p}<$ $.002)$, y SaO2 $(\mathrm{F}=9.94, \mathrm{p}<.000)$ con el SAHOS. Los parámetros en los que no hubo un incremento significativo en la severidad del IAH fueron la talla $(\mathrm{F}=$ $2.48, \mathrm{p}=.061)$ y el perímetro abdominal al nivel de la cintura $(\mathrm{F}=.802, \mathrm{p}<.498)$. Conclusiones: Los factores antropométricos (peso, IMC, PC, Epworth y SaO2) pueden ser utilizados como predictores de la severidad del SAHOS. Dentro de los valores antropométricos que mejor predicen la severidad del SAHOS son el IMC y la saturación basal de oxígeno.

\section{Efecto del CPAP sobre el riesgo cardiovascular en población con apnea obstructiva del sueño y diabetes mellitus 2. Resultados preliminares}

Canseco-Raymundo AR, ToledoVarela M, Castorena-Maldonado AR, Anaya-Ramírez SN, Galicia-Polo ML, Torre-Bouscoule L

Instituto Nacional de Enfermedades Respiratorias Ismael Cosío Villegas

Introducción: La diabetes mellitus 2 (DM2), es considerada un factor de riesgo para enfermedad cardiovascular. Diversos mecanismos de la apnea obstructiva del sueño se han visto involucrados en la generación de enfermedad cardiovascular. Diversas herramientas se han desarrollado para cuantificar el riesgo cardiovascular. El riesgo estimado de la cohorte Framingham, predice el riesgo de eventos cardiovasculares a diez años. El efecto del CPAP sobre el riesgo cardiovascular permanece incierto. Hipótesis: El tratamiento con CPAP en los pacientes con DM2 y apnea obstructiva del sueño disminuirá el riesgo cardiovascular, medido por Framingham. Objetivo: Evaluar el efecto de la CPAP sobre el riesgo cardiovascular medido por Framingham en una población con apnea obstructiva del sueño y diabetes mellitus 2 ajustada a un régimen de actividad física y nutrición. Material y métodos: 25 pacientes del primer nivel de atención medica, mayores de 20 años de edad con apnea del sueño, IMC $\geq 30 \mathrm{~kg} / \mathrm{m}^{2}$ y diabetes mellitus 2, fueron aleatorizados a recibir CPAP óptimo o CPAP sham por 12 semanas. Se realizaron mediciones de tensión arterial, colesterol y colesterol-HDL de forma basal y 12 semanas después, y se estableció un plan de dieta, ejercicio y tratamiento médico durante todo el estudio. Resultados: En pacientes con uso de CPAP óptimo $(n=14)$, el CPAP redujo el riesgo cardiovascular global, la tensión arterial sistólica y el colesterol total, sin beneficio en la medición de colesterol HDL. Se encontró un aumento del riesgo cardiovascular global y tensión arterial sistólica en el grupo CPAP sham ( $\mathrm{n}=$ 11), así como disminución de las cifras totales de colesterol y sin beneficio en la fracción HDL. Conclusiones: El CPAP tiene una tendencia a disminuir el riesgo cardiovascular global en la población con apnea del sueño y diabetes mellitus 2 .

\section{Efecto de la estimulación magnética transcraneal en el síndrome de piernas inquietas $y$ movimientos periódicos de extremidades}

Arana-Lechuga DY, * CervantesGonzález Al, * García-Toribio MG, * Sánchez-Escandón $O,{ }^{*}$ VelázquezMoctezuma J, ${ }^{\ddagger}$ Shkurovich-Bialik $P^{*}$

*Centro Médico ABC, ${ }^{\star}$ Clínica de Trastornos del Sueño UAM

Introducción: El síndrome de piernas inquietas (SPI) es un trastorno neurológico frecuente, asociado a deficiencia de hierro y disfunción dopaminérgica, caracterizado por incomodidad en las piernas y necesidad por moverlas con exacerbación nocturna. El trastorno de movimientos periódicos de las extremidades (TMPE) es una importante comorbilidad; ambos provocan mala calidad del sueño. La estimulación magnética transcraneal (EMT) es un método no invasivo de 
neuroestimulación que genera un flujo de corriente suficiente para modular la excitabilidad cortical y se emplea cada vez más como tratamiento de distintos trastornos neurológicos y neuropsiquiátricos. Hipótesis: La EMT a bajas frecuencias podría ser útil en patologías con hiperexcitabilidad cortical. Objetivos: Describir los posibles efectos terapéuticos de EMT en SPI y TMPE. Material y métodos: Se presenta un paciente masculino de 33 años que acude a la Clínica de Trastornos de Sueño por insomnio, tras la valoración clínica se diagnostica SPI y se confirma con polisomnografía (PSG). El paciente fue sometido a EMT repetitiva de baja frecuencia durante 10 días consecutivos en corteza motora primaria izquierda realizada en el Servicio de Neurofisiología Clínica del Hospital ABC, Santa Fe, con un equipo Magstim Super Rapid. Resultados: Con la escala de puntuación de intensidad de síntomas (IRLS) el puntaje inicial fue de 25 (severo), al finalizar su tratamiento de 7. La PSG pretratamiento reveló 134 movimientos de extremidades e índice de 26.3 movimientos/hora de sueño; la PSG postratamiento reveló 88 movimientos periódicos de extremidades con índice de 10.5 movimientos/hora de sueño; con mejoría en la arquitectura del sueño. El paciente reportó mejoría en la incomodidad de sus piernas y con ello del insomnio. Conclusiones: La EMT de baja frecuencia podría resultar una alternativa no invasiva, efectiva y mejor tolerada para estos pacientes, siendo necesaria la realización de ulteriores estudios con muestras mayores y seguimiento a largo plazo.

\section{Efectividad del tratamiento cognitivo conductual en un grupo de pacientes con insomnio crónico}

\section{Jiménez-Correa U, Marin-Agudelo A, Verde-Tinoco S, Ramos-Alvear M, Santana-Miranda R, Galicia-Polo ML}

Clínica de Trastornos del Sueño, División de Investigación, Facultad de Medicina, UNAM

Introducción: El tratamiento cognitivo conductual (TCC-I) es la primer opción de tratamiento para el insomnio; sobre todo para evitar los síntomas de tolerancia y dependencia provocados por el mal uso de los fármacos hipnóticos. Material y métodos: Es un estudio prospectivo en el que se incluyeron 49 pacientes con insomnio crónico, $61.2 \%$ del sexo femenino, edad promedio de 51.9 años (entre 20 y 82 años), 30.6\% de ellos con insomnio primario y $69.4 \%$ con insomnio comórbido de la Clínica de Trastornos del Sueño de la Facultad de Medicina de la UNAM. Se les aplicó un programa de TCC-I y se hizo un seguimiento mensual durante 3 meses. Se usó el análisis de varianza de una vía y la prueba $\mathrm{C}$ de Dunnet para las comparaciones múltiples. Resultados: Se identificó una disminución significativa de la latencia subjetiva al sueño (0.007), de las noches de insomnio por semana (0.001), despertar prematuro (0.001), dificultad para levantarse (0.032), cansancio (0.001) y somnolencia (0.004); junto con un incremento significativo del tiempo total de sueño (0.001) y de la calidad subjetiva del sueño (0.001). Conclusiones: De acuerdo con lo indicado por la literatura, el TCC-I mostró una efectividad importante para controlar los síntomas, nocturnos y diurnos de insomnio crónico (primario y asociado a comorbilidad respiratoria durante el sueño).

Una nariz artificial disminuye la presión de salida en un dispositivo de presión positiva continua

Díaz-Santos GA, Torres-Fraga MG, Carrillo-Alduenda JL, Torre-Bouscoulet L, Pérez-Padilla JR, López-Escárcega E

Instituto Nacional de Enfermedades Respiratorias Ismael Cosío Villegas

Introducción: Los dispositivos de presión positiva continua generalmente se utilizan con humidificación para disminuir efectos adversos; sin embargo, éstos añaden costo. En la práctica clínica no es infrecuente que se utilicen narices artificiales como alternativa temporal, aunque agregan resistencia y su efecto sobre la presión de salida es desconocido.
Material y métodos: A un dispositivo de presión positiva continua (REMstar Philips Respironics) previamente calibrado y sin error entre la presión programada y la presión de salida, se colocaron 7 narices artificiales de marcas diferentes y la presión de salida se midió, desde 4 hasta $20 \mathrm{cmH}_{2} \mathrm{O}$ con un manómetro manual después de 2 minutos de estabilizarse el flujo de aire; la fuga intencional se mantuvo constante en todo momento y la fuga no intencional fue de $0 \mathrm{~L} / \mathrm{min}$. Resultados: El acuerdo entre la presión programada y la presión medida después de colocar la nariz artificial fue variable, con diferencias entre marcas y algunas con gran impacto sobre la presión de salida: INTERSURGICAL coeficiente de correlación intraclase (CCI) 0.95, diferencia de medias $(\mathrm{DM}) 1.3 \pm 0.4$ cm $\mathrm{H}_{2} \mathrm{O}$; ALTECH CCI 0.95, DM 1.4 $\pm 0.4 \mathrm{cmH}_{2} \mathrm{O}$; AIR CCI 0.98 DM $0.8 \pm$ $0.005 \mathrm{cmH}_{2} \mathrm{O}$; DRAGER CCI 0.97, DM $1 \pm 0.3 \mathrm{cmH}_{2} \mathrm{O}$; HUMID-VENT $2 \mathrm{~S}$ CCI 0.99 , DM $0.5 \pm 0.2 \mathrm{cmH}_{2} \mathrm{O}$; HUMIDVENT FILTER PED CCI 0.87, DM 2.3 $\pm 0.7 \mathrm{cmH}_{2} \mathrm{O}$; TWINSTAR DRAGER CCI 0.45, DM $5.5 \pm 2 \mathrm{cmH}_{2} \mathrm{O}$; además, se encontró una correlación directamente proporcional entre la presión programada y el $\Delta$ de presión expresada en $\mathrm{cmH}_{2} \mathrm{O}$, pero inversamente proporcional cuando se expresa como \% de pérdida. Conclusiones: La nariz artificial disminuye la presión de salida de un dispositivo de presión positiva continua en forma variable; los factores que afectan dicho cambio son la marca del filtro y la presión programada.

\section{Síntomas asociados a trastornos del dormir en pacientes de tercer trimestre de embarazo. Estudio piloto}

\section{Que-Salinas MI, Reyes-Zúñiga M, Torre-Bouscoulet $L$}

Instituto Nacional de Enfermedades Respiratorias Ismael Cosío Villegas

Introducción: Los cambios fisiológicos durante la gestación modifican el patrón 
y la duración del sueño, algunos cambios predisponen el desarrollo de trastornos del dormir. El ronquido habitual es reportado en $16 \%$ de las embarazadas, se ha identificado como factor de riesgo independiente para hipertensión gestacional (OR 2.03; p < 0.05) y restricción del crecimiento intrauterino (OR 3.45; $\mathrm{p}<0.01$ ). Períodos de sueño $<7$ h al día se asocian con diabetes gestacional (OR 11.7, IC 95\% 1.2-114.5). El insomnio se presenta hasta en el 54\%. La prevalencia de síndrome de piernas inquietas (SPI) es de 26 a $32 \%$, puede estar asociado a incremento del riesgo cardiovascular. No hay información en la literatura de las características de sueño de pacientes mexicanas. Objetivo: Identificar la frecuencia de síntomas asociados a trastornos del dormir de pacientes mexicanas de tercer trimestre de embarazo y describir las características autorreportadas de sueño. Material y métodos: Se diseñó un estudio de sección transversal en el que pacientes voluntarias en seguimiento de embarazo del Hospital Militar de Especialidades de la Mujer y Neonatología (HMEMYN) contestaron un cuestionario de hábitos de sueño, la Escala de Somnolencia de Epworth, ISI, HADS, síndrome de piernas inquietas y Berlín, y del expediente obstétrico se obtuvo peso y talla. Resultados: Se valoró un total de 72 pacientes, la edad promedio fue de $28 \pm 5.3$ años, 29 $(40.3 \%)$ tenían un IMC mayor a $30 \mathrm{~kg} /$ $\mathrm{m}^{2}$; las horas de sueño diario promedio fue $7.4 \pm 1.37 \mathrm{~h}$; el insomnio fue identificado en 29 (40.3\%) pacientes, SPI en $13(18.1 \%)$ de ellas, el ronquido habitual fue reportado por 13 (18.1\%) pacientes y apneas presenciadas por 5 (6.9\%); de acuerdo con la calificación de Berlín 15 (20.8\%) pacientes tuvieron riesgo alto para SAOS. HADS mayor a 7 en ansiedad se identificó en 14 (19.4\%) pacientes y en depresión en 11 (15.2\%). Conclusiones: Los trastornos del sueño, como insomnio y síndrome de piernas inquietas, al igual que síntomas como el ronquido habitual durante el tercer trimestre de embarazo, en la población estudiada, parecen tener una frecuencia similar a la reportada en la literatura.
Ventilación mecánica no invasiva guiada por manometría esofágica en una paciente con hipoventilación obesidad y apnea obstructiva del sueño

Cámara-Fernández J," Siroti $C, \neq$ Montiel Gs
*Instituto Nacional de Enfermedades Respira- torias Ismael Cosío Villegas, ^Hospital Cetrángolo Buenos Aires Argentina, §Sanatorio Municipal Dr. Julio Méndez Buenos Aires Argentina

Introducción: La ventilación mecánica no invasiva (VNI) es una parte muy importante del tratamiento del síndrome de hipoventilación obesidad (SHO). Presentamos el caso de un paciente con SHO y apnea obstructiva del sueño grave en quien la programación del ventilador estuvo basada en mediciones de mecánica ventilatoria. Caso clínico: Mujer de 56 años de edad, con IMC de $51 \mathrm{~kg} / \mathrm{m}^{2}$, $\mathrm{PaCO}_{2}$ de $52 \mathrm{mmHg}$ y $\mathrm{HCO} 3$ de $29 \mathrm{mEq} / 1$, a la cual en posición sentada se le colocó un balón esofágico. Previa instilación de la fosa nasal con lidocaína al $2 \%$ se progresó el catéter hasta la posición esperada e insufló con $0.5 \mathrm{~mL}$ de aire, verificando la correcta posición mediante técnica de Baydur. Para medir los flujos inspirados, espirados y la presión en la vía aérea se utilizó un neumotacógrafo de presión diferencial. Tanto el sensor de flujo como el catéter balón fueron conectados al monitor de mecánica ventilatoria FluxMedGrE, MBMed. Se realizaron las siguientes mediciones con la paciente en decúbito supino: el volumen corriente, flujo respiratorio, presión de vía aérea, presión esofágica (Pes), presión transpulmonar teleespiratoria (PLes), la relación volumen corriente/swing de presión esofágica (VC/ PesSwing) y el producto presión tiempo total (PTP). Se observó que la Pes era de $25 \mathrm{cmH}_{2} \mathrm{O}$, la PLes de $-25 \mathrm{cmH}_{2} \mathrm{O}$, el PTP elástico de 11.2 y el PTP resistivo de 1.8. Con base en la información obtenida se tituló la EPAP en $18 \mathrm{cmH}_{2} \mathrm{O}$, con el objetivo de obtener una PLes cercana a 0
$\mathrm{cmH}_{2} \mathrm{O}$, incrementar el VC y la relación VC/PesSwing obteniendo un descenso del PTP total a 4.27. Dado el poco componente resistivo del PTP, se necesitó poca presión de soporte $\left(5 \mathrm{cmH}_{2} \mathrm{O}\right)$. Con dichos valores se obtuvo un índice de apnea hipopnea residual de 5 y la corrección en los valores de $\mathrm{CO}_{2}$ diurno (41 $\mathrm{mmHg}$ ). Conclusiones: La utilización de manometría esofágica permite ajustar las presiones de VMNI en pacientes con SHO de manera más objetiva y ajustadas a las necesidades mecánicas de estos pacientes.

\section{Prevalencia y correlato clínico de las alteraciones del sueño en pacientes con enfermedad de Parkinson: Un estudio polisomnográfico}

\section{Alatriste Booth-VJ, Rodríguez- Violante M, Camacho-Ordoñez A, Cervantes-Arriaga $A$ Instituto Nacional de Neurología
y Neurocirugía}

Introducción: Los trastornos del sueño en la enfermedad de Parkinson son de los síntomas no motores más comúnmente encontrados con una prevalencia de entre el 60 y 90\%. Los instrumentos clínicos se usan frecuentemente para diagnosticar dichos trastornos; sin embargo, el estudio polisomnográfico es el estándar de oro. Objetivos: Determinar la prevalencia de los trastornos del sueño en enfermedad de Parkinson por medio de polisomnografía, así como evaluar los factores clínicos asociados. Material y métodos: Se incluyó un total de 120 pacientes con diagnóstico de enfermedad de Parkinson. Se recolectaron datos demográficos y características de la enfermedad y se aplicaron las escalas de UPDRS (Unified Parkinson's Disease Motor Rating Scale) así como SCOPA para los trastornos del sueño. A todos los pacientes se les realizó un estudio polisomnografico. Resultados: $98 \%$ de los pacientes tuvieron estudios polisomnograficos con resultados anormales. Más de la mitad de los pacientes presentan SAHS, el cual 
se asoció con mayor frecuencia a mayor edad y mayores índices de masa corporal ( $\mathrm{p}=0.049$ y $\mathrm{p}=0.016$ ) respectivamente. El $37.5 \%$ de los pacientes presentó trastorno conductual del sueño MOR, el cual se asoció con una mayor edad de aparición $(\mathrm{p}=0.001)$, inicio de los síntomas motores del lado izquierdo (p $=0.047)$ y a mayores dosis de levodopa (0.016). No se encontraron factores asociados a movimientos periódicos de las extremidades. Conclusiones: La apnea obstructiva del sueño y el trastorno conductual del sueño MOR son los trastornos del sueño más frecuentemente asociados a la enfermedad de Parkinson.

$\triangle$ Correspondencia:

Dr. José Luis Carrillo-Alduenda,

Presidente de la Academia Mexicana de Medicina del Dormir.

Correo electrónico: jlcarrillo14@hotmail.com 